\title{
UNA VISIÓN HOLÍSTICA DE LA NATURALEZACULTURA: EL COSMOTEANDRISMO EN LOS GLIFOS DE LAS CIUDADES PREHISPÁNICAS DE HABLA NAHUATL
}

\author{
(A holistic view of nature-culture: Cosmotheandrism in the \\ glyphs of pre-Hispanic towns of Nahuatl language)
}

Francesca Zunino ${ }^{1}$

(Università di Módena e Reggio Emilia, Italia)

\begin{abstract}
The issues concerning the overcoming of the false dichotomy between the two apparently opposite concepts of "nature" and "culture" represent the central and most urgent core of both Ecolinguistics" integrated standpoint and global society's general discourse. In order to eclipse this separation, which is the most damaging for the anthropoecosystem among the basic contradictions of our social praxis (Ibid.), our cognitive, discursive and performative perspective needs a radical change. This transformation shall involve the use of new terms and references that holistically illustrate the consubstantial inter-relation between the "human" and the "natural" being - that is, of all planet's being -, such as the "natureculture" neologism. The present analysis aims at applying the triadic perspective of cosmoteandrism - the ecosophic integration among the cosmic, the human and the devine in the eprception of reality, of being and of the planet - to Ecolinguistic studies, investigating how the union among the anthropological, natural and teological dimensions is represented in the discourse of the Nahuatl speaking populations of pre-Hispanic Mexico; and specifically, in the ethnotoponymy and in the visual representations of the glyphs indicating the pre-Columbian cities and geographic locations. A new diachronic philosophically and ecologically integrated construction of narratives

1. Doutora em Ciências Humanas (Modena e Cheltenham), com uma tese em ecolinguística, mestre em Questões Ambientais na América Latina (Londres). Graduada em Línguas e Culuras Modernas (Turim). Área privilegiada de pesquisa: línguas e culturas ameríndias do México.
\end{abstract}


and identities, a new "greenspeak" or better a new "ecological speaking" which shall be applicable, alternative and constructive (following the four macro-strategies classification of discourse construction, van Leeuwen y Wodak, 1999), must also start with the search and use of languages and imageries originating in non-Eurocentric world visions, and therefore less prejudiced by the harmful dualism that characterizes the Standard Average European languages), exported and used since the colonial period.

Key words: Holims, Natureculture, Cosmoteandrism, Integration, PreHispanic, Nahuatl.

\section{RESUMO}

O núcleo central e mais urgente da Ecolinguistica, bem como do discurso geral e da sociedade global, é superar a falsa dicotomia entre os conceitos de «natureza» e «cultura», o mais prejudicial ao ecossistema antropológico entre as contradições básicas da nossa práxis social. Para alcançar este objetivo, a nossa perspectiva cognitiva, discursiva e pragmática necesita de uma mudança radical, envolvendo o uso de novas palavras e referências que explicam de forma holística a inter-relação inerente entre «humano» e de ser «natural» - isto é, todo o ser do planeta - como o neologismo «naturezacultura». Nesta análise, pretendemos aplicar a perspectiva triádica cosmoteandrismo - ecosophic - integração entre o cósmico, o humano e o divino, na percepção da realidade do ser e do ser humano - aos estudos da Ecolinguistica, investigando a união entre as dimensões antropológica, natural e teológica no discurso de povos da língua nahuatl do México pre-hispânico, mais especificamente $n$ etnotoponimia e nos aeroglifos dos nomes de cidades e localizações geográficas pré-colombianas. A construção de novas narrativas e identidades, integradas filosófica y ecolinguísticamente, um novo "greenspeak", um novo "discurso ecológico" aplicável, alternativo e construtivo, deve começar também pela busca e uso da linguagem, bem como das imagens provenientes de visões de mundo não eurocêntricas e não é afetado pela dualidade destrutiva que caracteriza as línguas europeias, exportadas e utilizadas desde os tempos coloniais.

Palavras-chave: holismo, 'naturezacultura', cosmoteandrismo, integração, pré-colombiano, nahuatl. 


\section{Introducción}

Las cuestiones que aquí se plantean son: ¿cómo se representaba la visión cosmogónica del hombre y de su realidad en el discurso de las poblaciones de habla nahuatl? ¿Había separación antropocéntrica ó interrelación entre las dimensiones teológica, antropológica y ecológica? De darse esta unión, ¿hay algún elemento que podría aplicarse hoy para una extensión de la ecosofía y para un discurso global más biocéntrico, que el etno-ecosistema necesita para la propia sobrevivencia del sistema Tierra? Se analiza un caso de posible integración de los espacios humanos, naturales y divinos en la expresión comunicativa, semiótica y multitextual de los pictogramas nahuatl del México prehispánico, los glifos de los nombres de algunas ciudades.

Este estudio enmarca en las más recientes investigaciones de ecolingüística, y trata de integrar las perspectivas teóricas y analíticas de esta área "transdisciplinaria" (Papst, 2003), con estudios de etnoecolingüística comparada - la hispanística histórica y las investigaciones sobre el nahuatl prehispánico. La lingüística aplicada, y todas las disciplinas de la comunicación, y las humanísticas en general - también algunas "científicas" - pueden adoptar un enfoque más holístico, cuyo marco general es la perspectiva de la ecolingüística. Fortaleciendo los análisis lingüísticos con el punto de vista ecológico y siguiendo la idea de Lingüística Ecosistémica, o la de Ecología de la Interacción Comunicativa de la escuela de Brasilia (Couto, en prensa) puede lograrse una visión más integrada. Nuestra perspectiva cognitiva, discursiva y pragmática necesita de un cambio radical, que supere la falsa dicotomía entre los conceptos de "naturaleza” y "cultura" (Bang y Døør, 1993), involucrando nuevas palabras y referencias lingüísticas, visuales y semánticas que ilustren holísticamente la interrelación consustancial entre el ser "humano" y el ser "natural", y de todo el ser del Planeta - como el neologismo "naturalezacultura" (Latour, 1998: 18-21; Döring, 2003: 198). Se necesitan investigaciones que involucren varios niveles comunicativos: del léxico, de las imágenes, de los signos, del discurso en su conjunto y su uso, a menudo retórico; los procesos de creación, repetición y 
trasmisión del significado a través de la praxis comunicativa, puesto que el lenguaje construye activamente la realidad, y la gramática refleja al mundo; la reconsideración de las identidades socio-ambientales o naturales-culturales de las componentes "humanas" y "naturales" del etno-ecosistema (con respeto a la contraposición antropocéntrica), de la imaginería semántica de los universos comunicativos, y de la geopoética (Ainsa, 2006) de los espacios de naturalezacultura; y finalmente, de la globalización y glocalización y sus recaídas planetarias.

Este estudio aborda temas de etnolingüística y de etnotoponimia a nivel ecolingüístico (Couto, 2007: 250-259), analizando los glifos de los topónimos precolombinos, para individuar si y cómo la unión entre las dimensiones antropológica, natural y teológica está representada en el discurso de las poblaciones de habla nahuatl del México prehispánico. Se considera el rol del lenguaje y de la comunicación en el origen y desarrollo de los problemas socio-ambientales, pero también el de la pesquisa lingüística como fuente de posibles soluciones ideológicodiscursivo-prácticas alternativas. Por ende, se acudió a metodologías, basadas también en la ecolingüística como análisis ecocrítico y análisis crítico del discurso (Glotfelty, Fromm, 1996; Fill, Mühlhäusler, 2001; Fairclough, 2003; Alexander, 2009; Fill, 2010) y "explanatory critique" (Fairclough, 2001: 125); al análisis semiótico de la "retórica de la imagen" (Barthes, 1964).

De hecho, los glifos son signos gráficos, pero también pictogramas e iconogramas, multitextos - como toda forma de comunicación y, este mismo ensayo -; a los estudios históricos del discurso (Wodak, 2001; Jäger, 2001). La construcción actual de la ontología psico-neuro-cognitiva del ser, globalizada y homologada, puede cambiar hacia una visión más sostenible, sistémica, compleja (Morin, 1992), cross-cultural, crítico-dialógico-dialéctica (Bang et al., 2008) y propositiva, si superamos el eurocentrismo dualista antropocéntrico y destructor que caracteriza las lenguas Standard Average European (Whorf, 2000), exportadas con la colonización, buscando otras construcciones diacrónicas, narraciones e identidades filosófica y ecolingüísticamente integradas. Este nuevo "greenspeak" (Harré et al., 1999), "habla ecológica" alternativa y constructiva 
(van Leeuwen, Wodak, 1999), podrá hallarse estudiando lenguajes e imaginerías de "otras" tradiciones y culturas, pasadas y presentes, biocéntricas y ecológicamente equilibradas. La perspectiva crosscultural de interacción comunicativa, aunque sin contacto directo, entre distintas culturas y Weltanschauungen, que también pertenece a la ecolingüística, es una herramienta para superar las contradicciones básicas de la praxis ideológica y social occidental. De hecho, los cambios en los esquemas conceptuales y las narrativas mitopoiéticas (Bowers, 2007: 6; Pennycook, 1994), la "transculturation" (Pratt, 1992) que empezaron tras la "moderna" (Dussel, 2000) llegada de los europeos a las Américas (Zunino, 2011), con la exportaciónimportación de identidades, ideas y cosmogonías socio-ecológicas - y sus relativos prerrequisitos culturales (Silverstein, 1977) -, continúan en el postmoderno glocal. La etapa actual de la historia de la mentalidad (Hermanns, 1995 en Jung, 2001:271) o historia lingüística del discurso (Jung, 2001:270), es un corte sincrónico en la corriente discursiva, en el flujo de la semogénesis (Hallyday, 2001: 179). De ahí surgen nuevos análogos y metáforas para enmarcar una realidad de equilibrio ecológico y los significados de sus términos, alternativa a la de quienes en pasado, desconociendo los límites de la capacidad de carga del socio-ecosistema perpetuaron errores y silencios.

\section{Cosmoteandrismo y panenteismo ecológicos, y el nahuatl prehispánico}

Se alude aquí a la perspectiva triádica del "cosmoteandrismo" de Panikkar (1994) - es decir, la integración entre lo cósmico, lo humano y lo divino en las percepciones de la realidad, del ser y del ser humano - en un sentido puramente ecosófico, como aporte adicional a la ética de la ecología profunda (Naess, 1973). Cabe subrayar que el cosmoteandrismo que se aplica en este estudio, se aparta del elemento cristiano de Panikkar, cuyo punto de partida es la trinidad del catolicismo. Sin embargo, el cosmoteandrismo rechaza la visión dualista y el monoteísmo monolítico, promoviendo el pluralismo, el 
diálogo interreligioso y la hermenéutica comparativa - Panikkar, de madre española y padre de la India, siempre buscó los nexos entre las ideas filosóficas, culturales y religiosas del este y del oeste, y entre la ciencia y la literatura. Las etapas de la historia del hombre que Panikkar define cosmocentrismo, antropocentrismo y cosmoteandrismo, pueden ajustarse a las de antropocentrismo destructivo - biocentrismo conservativo de la ecología profunda. Utilizaremos así la idea, sugestiva y muy incluyente, del cosmoteandrismo para indicar a nivel macroscópico la interconexión entre los planes del cielo, de la tierra y del inframundo, entre dioses, humanos y naturaleza, en la concepción y representación de la realidad por parte de los mexicanos prehispánicos de habla nahuatl.

Otra concepción importante para la ecosofía general - y para nuestros análisis - es el "panenteismo" de raigambre cristiana y ecológica de Boff (1993), quien propone superar el antropocentrismo de abolengo judaico-cristiano y volver a una concepción mística y sagrada de la naturaleza, para una nueva democracia ecológico-social. Según Boff, no todo es dios, pero dios está en todo: de ello surge una nueva espiritualidad integradora y holística. El panenteismo puede extenderse en sentido interreligioso, diacrónico-histórico y transcultural, y aplicarse a nuestra investigación en paralelo al cosmoteandrismo, para analizar el entrelazamiento de dioses, hombres, flora, fauna y elementos del cosmos en la cultura azteca precolombina.

En este ensayo nos referimos al idioma nahuatl clásico, hablado en la capital azteca y cristalizado con letras y ortografía latinas en la época de la conquista y del siglo XVII, debido a Bernardino de Sahagún con su Historia general de las cosas de Nueva España redactada gracias a informantes indígenas entre 1540 y 1585 en náhuatl y español, donde se encuentran amplios testimonios de 'las cosas divinas, humanas y naturales' de los nahuas; a Andrés Olmos (Arte de la lengua nahuatl o mexicana, 1547), Alonso de Molina (Vocabulario de la lengua mexicana y castellana, 1571), y Horacio Carochi (Arte de la lengua mexicana, 1645, ed. Macazaga Ordoño, 1979). De hecho, según Hasler Hangert (1995) antes de la caída del imperio Mexica en 1521, el nahuatl se hablaba en gran parte de lo que 
hoy es México y, hasta Nicaragua, siendo su variante de Tenochtitlan la que había sido impuesta como la única forma correcta. Sucesivamente el casi aislamiento de distintas poblaciones de habla nahuatl impulsó la evolución divergente de las variantes locales, más o menos alejadas del original. Solo conocemos el nahuatl clásico a través de los documentos de la primera época de la colonia.

Hay que resaltar también, que América Latina (y todo el continente), es una macro-área sumamente importante para los estudios de lingüística y comunicación, así como para un sinnúmero de temas de investigación, sobre todo transdisciplinarios, por su enorme etno- y biodiversidad (Halffter, 1992), y por ser un crisol de ideas, reflexiones e influencias remotas y contemporáneas, desde cuando poblaciones asiáticas de Homo sapiens llegaron más de once mil años atrás (Mann, 2005:17) - antes de la revolución neolítica y la invención de la agricultura en Eurasia. Asentadas en el istmo mesoamericano, en el Pleistoceno tardío (Dillehay, 2000; Dull, 2008:323), crearon sus extraordinarias técnicas y prácticas agrícolas en total independencia, hace quizás diez siglos ${ }^{2}$. La revolución agrícola mesoamericana pudo darse gracias a una gramínea silvestre del género Zea (Bartra, 2003:219-250; Dull, 2006:357-358), el "teocinte" o "teosinte" - castellanización del nahuatl, "teocentli" o "teoxintli", "maíz divino". Gracias a la polinización cruzada y selección, o quizás a una mutación, los mesoamericanos crearon las muchísimas variedades del maíz, que cultivaron a menudo en conjunto con frijol y calabaza (las tres hermanas o la milpa, un agroecosistema todavía en uso, rescatado hoy por altamente sostenible y ecológico, símbolo de resistencia cultural a las empresas transnacionales). El maíz y sus innumerables representaciones pictóricas, narrativas, mitológicas pasadas, y sincréticas presentes, es el protagonista de los discursos cosmogónicos precolombinos y también modernos y contemporáneos, atributo esencial del nacimiento de los dioses y de la humanidad (y de la ontología de todo el ser), ejemplo sumo de la unión holística y cosmoteándrica en cada expresión de las culturas que se forjaron gracias

2. algunos investigadores hasta vislumbran una revolución neolítica más en la historia del hombre, independiente de las otras, que habría ocurrido a los pies de los Andes y en una época anterior a la revolución del maíz (Mann, 2005:18). 
a él, y hasta lo que hace que la mayoría de los pueblos latinoamericanos se reconozcan como "hombres de maíz" (Asturias, 1949) 3

Como sugiere do Couto (2007:250-251), el estudio de la "microtoponimia", de los nombres que las comunidades han dado a los aspectos de su medio ambiente inmediato, corresponde aproximadamente a la etnotoponimia: los etnotopónimos, están intrínsicamente relacionados con una comunidad, de la que forman parte integrante. En este ensayo se analizan micro-etnotopónimos mexicanos prehispánicos no tanto para averiguar las "huellas de la historia de un lugar ... [para] identificar la pérdida de una lengua .... la transformación de un paisaje" (Valenzuela Valdivieso, 2010:18), sino porque "los topónimos, a su vez, son descriptivos de los aspectos culturales de las personas y las sociedades. Hay nombres que están relacionados con la cosmovisión, la religión, las tradiciones y la historia ...” . Lo que interesa aquí es el paisaje cultural y natural más amplio, junto con sus referencias y reflejos ecológicos, lingüístico-metafóricos, teológico-mitológicos, cosmogónico-totémicos, botánicos, zoológicos y de identidad antropológica desde un punto de vista "milenario" (León-Portilla, 1983; 2009): los lugares reúnen historia y autobiografía, pero también son puntos de encuentro privilegiados para humanos, plantas, animales y espíritus (Couto, 2007:254). En esta visión enmarca la tradición del origen mítico de las ciudades de la Mesoamérica precolombina: según Margaloni Kerpel (2011), ellas reflejan simbólicamente los fundamentos del pensamiento tradicional indígena, el orden divino de la construcción de la Tierra como un ser vivo desde el principio, y la erección del árbol cósmico que está entre los cielos y la tierra y permite que el sol y su recorrido den inicio al tiempo.

Una investigación extensa y profundizada de los glifos prehispánicos, aunque muy difícil debido a la gran complejidad de las cosmovisiones de las culturas que involucraría, sería extremadamente

3. Sin olvidarse que, debido a la fuerte producción de variedades genéticamente modificadas, y a la mercadotecnia agresiva, hoy el maíz está en grave peligro, así como el ecosistema en que se le cultiva y las personas y los animales que lo consumen - ver por ejemplo Greenpeace, 2003 y otros documentos en la página http://www.greenpeace.org/international/en/campaigns/agriculture/problem/ genetic-engineering/ge-agriculture-and-genetic-pol/ge-crops/maize/. 
interesante y necesaria en el marco de la ecolingüística, y también para ampliar el universo de muchas áreas de la lingüística, puesto que los límites de la comunicación mixta de las lenguas de la Mesoamérica son sumamente extensos, y la diferencia entre iconografía y palabra no corresponde a la nuestra, ya que "en tiempos precolombinos ... El verbo y la imagen se vinculaban estrechamente en la producción del sentido sin que el discurso pictórico se sometiera del todo, sin embargo, a la 'tiranía' de su señoría la palabra. La imagen producía un sentido específico con recursos propios que si bien se podía 'leer' parcialmente y reducir a palabras no se petrificaba en un texto verbal determinado. Según el género pictórico, se establecía una relación específica entre el sentido referido y el sentido producido por la imagen; entre la historia y el discurso, entre los niveles de superficie y las estructuras profundas de la configuración pictórica. La mímesis icónica, el simbolismo ideográfico y la mediación fonética se conjugaban con el tamaño, el trazo, la posición, los colores, la tensión espacial de las formas y su composición para generar un sentido sensible, en parte infraliminal." (Johansson Keraudren, 2001:69).

Finalmente, cabe insistir con Klein (2004:29) en que los análisis iconográficos de Mesoamérica, aunque obviamente sesgados, remontan a los años inmediatamente posteriores a la Conquista, y al contacto directo entre los primeros iconógrafos y las poblaciones nativas. A finales de 1800 el lingüista E. G. Seler entendió que "muchas de las formas pictóricas eran, en efecto, símbolos o metáforas visuales de conceptos clave que podían decodificarse", mientras el historiador del arte E. Panowsky, "situaba la imagen en el contexto de las ideas, valores y tradiciones del tiempo y el lugar de su creación, etapa altamente especulativa del proceso iconográfico a la que llamó iconología” (p. 30). Hoy la semiótica ayuda la iconografía-iconología, junto con la lingüística, la historia del arte, la historia y otras disciplinas, a reunir las diferentes perspectivas de análisis de las imágenes en una única y múltiple. Actualmente, es grande "...la necesidad de distinguir en la iconografía mesoamericana entre los íconos que remiten a la percepción sensorial de un objeto y los que apuntan a la aprehensión conceptual del mismo. Los primeros se refieren a la apariencia visual atribuible 
a un objeto, independientemente de su naturaleza real o imaginaria. Los segundos, en cambio, se refieren a los atributos del objeto: derivan de su representación mental. ... ¿ Por qué las figuras cósmicas tienen tal abundancia de calificadores? Porque responden simultáneamente a principios nomológicos y a funciones cósmicas específicas. En su sentido más abstracto, representan una ley divina general; en contraste, señalan con sus múltiples calificadores cada una de sus operaciones en la dinámica del universo." (López Austin, 2006: 93-95).

\section{Análisis}

Los glifos ${ }^{4}$ estudiados se encuentran en la la Matrícula de Tributos y en el Códice Mendoza ${ }^{5}$, publicada en Nomenclatura geográfico, etimológica y jeroglífica, nombres geográficos de México (A. Peñafiel, $1885)^{6}$, que incluye 462 glifos y 39 láminas, editada en versión digital por el Aztec Place Name Glyphs Project de la Universidad de California, Berkeley ${ }^{7}$. La ubicación antigua y contemporánea de la mayoría de los lugares de la Matrícula se conocen gracias a Barlow (1949), ahora accesibles como mapa interactivo ${ }^{8}$. El presente estudio sigue la edición interactiva, que a su vez sigue fielmente la edición de Peñafiel; también utilizamos León-Portilla (1982), y el recurso digital Tetlacuilolli9 del CIESAS, México.

4. En realidad sería más apropiado denominarlos "compuestos glíficos”, puesto que están formados por dos o más glifos que contribuyen a dar sus significados.

5. El primero es uno de los códices mexicas de origen prehispánico, aunque elaborado o copiado entre 1522 y 1530 , que registra en hojas de papel amate tradicional y en forma pictográfica los tributos que las poblaciones del imperio mexica pagaban periódicamente a Tenochtitlán entre los siglos XV y XVI, y con descripciones muy poco posteriores escritas en español; la segunda parte del Códice Mendoza es una copia completa de la Matrícula, de 1535, sobre papel europeo. La Matrícula de Tributos se encuentra digitalizada en la Biblioteca Digital Mexicana (http://bdmx. $\mathrm{mx} /$ manuscritos_tributos.php).

6. Consultable en la página http://archive.org/details/nombresgeogrfic00fomegoog.

7. http://oldweb.geog.berkeley.edu/ProjectsResources/Glyphs/Home.html.

8. http://oldweb.geog.berkeley.edu/ProjectsResources/Glyphs/Keys/ BarlowMapKey.html.

9. http://www.tetlacuilolli.org.mx/. 
Esta investigación, preliminar a un próximo desarrollo, se limita a cuatro glifos. El criterio de elección fue considerar compuestos glíficos que abarquen a nivel macroscópico las áreas de análisis de naturaleza y cultura, incluyendo un elemento principal de tipo vegetal, animal, y humano. Se escogieron así los glifos de Cuauhnahuac (1, fig. a), Huaxyacac (lám. fig. b).

Lámina 1. Los cuatro compuestos glíficos analizados

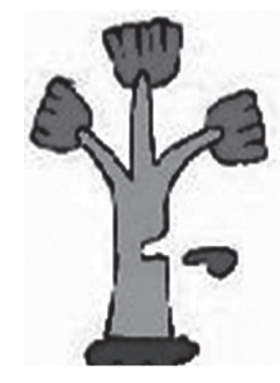

a. Cuauhinahuac o Cuauhnahuac

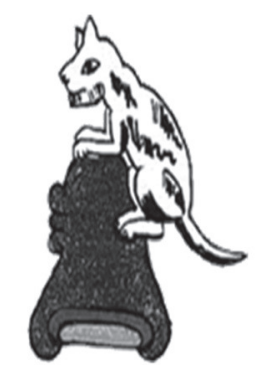

c. Izcuintepec

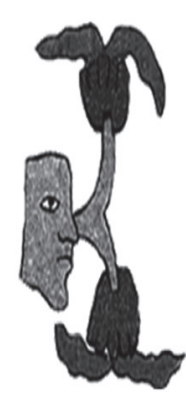

b. Huaxyacac

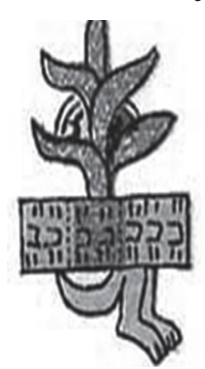

d. Acamiltzinco

(imágenes de http://oldweb.geog.berkeley.edu/ProjectsResources/Glyphs/Home.html) Huaxyacac (lámina 1, fig. b), Izcuintepec (lámina 1, fig. c) y Acamiltzinco (lámina 1, fig. d). Izcuintepec (lám.1, fig. c) y Acamiltzinco (lám. 1, fig. d). 
Cuauhinahuac o Cuauhnahuac (Matrícula, Lám. 24, fig. 1).Tiene nombre y glifo en parte figurativo y en parte ideográfico, presentando el signo árbol, que se lee "coahuitl" o "cuahuitl", con una abertura bucal anotada en el lado derecho del tronco y su relativo signo, símbolo e ícono con forma de vírgula a representar la palabra, el lenguaje, el acto de hablar. Este ícono, muy frecuente, se lee "nahua" y significa "cerca", "junto con" (Macazaga Ordoño, 1979:64), "adentro del rango auditivo", "a distancia de oído" (según Mc Cafferty ${ }^{10}$ la raíz /na:wa-/ significa "audible", "sonido claro",). "Cuahuitl" y "nahua", con la terminación locativa "c", se lee entonces "cuauhinahuac" y da la significación de "cerca del bosque", "junto a la arboleda", "lugar rodeado de árboles". ${ }^{11}$ Cuanto al uso de la imagen y de los glifos del árbol, hay que notar que Sahagún (1975, Lib. XI, Cap. VI, pp. 660665)." "12 reporta una gran cantidad de árboles, con sus usos, con frecuencia asociados a topónimos. En Cuauhnahuac vivió Juana de Zúñiga, esposa de Hernán Cortés ${ }^{13}$; el nombre actual, castellanizado con varias pronunciaciones progresivas eufónicas e incorrectas del original, a comenzar por Cortés, es Cuernavaca, capital del estado de Morelos.

Cabe resaltar que la aplicación de uno de los principales atributos humanos (la palabra) a un elemento no humano (el árbol), trasciende el dualismo entre lo que la sociedad occidental considera "cultural" y "natural". El árbol fue "para las tradiciones mesoamericanas la vía de unión entre el cielo y el inframundo" (López Austin, 1997:91), del que provenía todo el orden

10. http://www.famsi.org/pipermail/nahuatl/2009-November/001200.html.

11. Escribe Peñafiel: "Cuauhnahuac. -- Cuauh-nahuac. -- Quauhnahuac. La palabra... es mitad figurativa y mitad ideográfica, el signo árbol, coahuitl, con la terminacion nahuac expresada por una abertura bucal, y la vígula de la palabra, dan la significacion de "cerca del bosque," ó en la orilla de la arboleda. En el Anaglifo de Aubin hemos encontrado una singular abreviatura ó variante de Cuahnahuac: una cabeza de cuadrúpedo carnicero con tres cuernos formados de ramas de árbol y la vírgula cerca de la boca, que dan la misma significación.” http://oldweb.geog. berkeley.edu/ProjectsResources/Glyphs/Plate09/Cuauhnahuac.html.

12. Véase http://www.tetlacuilolli.org.mx/comentarioGlifo.php?id $=1235594083$

13. http://www.e-local.gob.mx/work/templates/enciclo/morelos/Municipios/17007a. htm. 
político y el poder, en cuyo tronco fluían las dos corrientes helicoidales de las fuerzas opuestas del inframundo y del cielo. Los mexica además representaban con la forma de una ceiba (Ceiba pentandra, Malvaceae) los 22 pisos del cosmos y del tiempo: los " 9 cielos altos" en el follaje y los 9 "lugares de la muerte" en las raíces, reservados a los dioses y donde existía el eterno presente y todas las posibilidades del ser, y las cuatro capas intermedias, reservadas a los seres terrenales, donde se desarrollaba cíclicamente el curso del mundo; y según un mito, dos de los dioses más antiguos e importantes de los mexica, Tezcatlipoca, dios de la oscuridad, y Quetzalcoatl ("Serpiente Emplumada”), dios de la luz, se transformaron en dos árboles. Hay que resaltar que la dualidad (la bipartición arriba/abajo, cielo/tierra, claridad/oscuridad), es tan solo aparente. De hecho, en las cosmovisiones mesoamericanas había una compenetración de niveles, concepciones y representaciones del cosmos - el cielo, la tierra y el inframundo - mucho más intricada de lo que puede entenderse superficialmente, siguiendo nuestra óptica occidental. Dabrowska (2008: 164) alega que "[en] las representaciones del cielo nocturno el mismo «diseño»... rellena el interior de la tierra.... [en] la imagen de la tierra devorando a un muerto, quien entra al mundo de los muertos, Mictlan, ... aquel lugar está pintado de la misma manera que el cielo nocturno. ... la incierta localización de Mictlan en el universo nahua —arriba y abajo- y su característica como un lugar creador, igual que Omeyocan [el lugar de la dualidad, donde vive Ometeotl, el dios dual, y de donde se originan todas las cosas manifiestas] permiten constatar que Mictlan y el cielo nocturno estaban percibidos de cierta forma como lo mismo. La presencia del «Mundo de los Muertos» tanto arriba como debajo de la tierra, explicaría ciertas imágenes gráficas y conceptuales, por ejemplo de la lámina 57 del Códice Borgia, en la que se representa a la «muerte» / la tierra en forma de cráneo que está devorando a un muerto sobre el fondo del cielo nocturno" .Esta visión multifacética, corresponde de cierta manera a nuestras ideas de cosmoteandrismo y panetenismo ecolingüísticos.

La segunda ciudad que examinamos es Huaxyacac (Matrícula , Lám. 46, fig.4). Su compuesto glífico representa una cara de hombre de 
cuya nariz brotan dos ramas de un árbol con follaje verde y seis vainas rojas. No cualquier árbol ("cuahuitl”): el signo, pictóricamente muy realista, corresponde al "huaxin", "nombre genérico [de] varios grupos de vegetales; entre ellos, el huaje y la calabaza" (Macazaga Ordoño, 1979:39). En este caso, se trata del guaje (Leucaena esculenta), una leguminosa comestible nativa de México que en el nahuatl moderno del estado de Guerrero se llama "tlapalwa:xin". Su glifo brota de la nariz ("yacatl") de la cara humana. "Yacatl" también significa "punta de algo", así que las raíces "huax", "yaca" y la terminación locativa "c", proporcionan la lectura fonética de "Huaxyacac", "en la cumbre de los huaxis" o "sobre la punta del lugar de los huaxis"14. Hoy esta ciudad se conoce como Oaxaca de Juárez, capital del estado de Oaxaca, dedicada al primer presidente mexicano de origen indígena y héroe nacional, Benito Juárez (1806-1872), nacido en un pequeño pueblo del estado. Una vez más hay una total compenetración entre los mundos humano y vegetal: la planta brota del hombre; la nariz es metáfora de cumbre en el paisaje. Además, la dimensión religiosa y teológica siempre está presente a cerrar y sellar nuestro círculo interpretativo: Broda (2009:41) sostiene que "Al estudiar la cosmovisión como visión estructurada en la cual los antiguos mesoamericanos combinaban de manera coherente sus nociones sobre el medio ambiente en que vivían, y sobre el cosmos en que situaban la vida del hombre, partimos de la ubicación de estas creencias en el mundo real. ... Simultáneamente, en la construcción de su cosmovisión, estos pueblos mezclaron conocimientos exactos con creencias mágicas acerca de la existencia y la actuación de los cerros que eran concebidos como seres vivos" y "los topónimos referidos a cerros, pueblos e infinidad de lugares

14. Escribe Peñafiel, confundiendo el guaje o Leucaena esculenta con la Acacia esculenta, y mencionando un "tepec" o "cerro" que no está: "Huaxyacac. -- Huax yaca c. -- Guaxaca. Una planta, signo del huaxin, bien caracterizada en la pintura, perteneciente á una leguminosa, Acacia esculenta, L., da la radical huax; implantada en la nariz, yacatl, de una cara, huax yaca; la terminacion $c$, equivalente á tepec está abreviada ó suprimida, pero expresada en el jeroglífico del Sr. Orozco y Berra: la lectura fonética dice : Huax yaca tepec, ó Huax yaca c. "En la cima de los huaxis," en la meseta que produce la Acacia esculenta, que es comestible." http://oldweb. geog.berkeley.edu/ProjectsResources/Glyphs/Plate13/Huaxyacac.html. 
sagrados del paisaje demuestran esa íntima fusión entre la percepción mítica y el paisaje real.”

El tercer glifo representa la ciudad de Izcuintepec (Lám. 15, fig. 6 de la primera parte del Códice Mendocino), reportado integralmente por Peñafiel ${ }^{15}$ ), la actual Santiago Ixcuintepec, estado de Oaxaca. Reproduce a un perro con manchas negras ("itzcuintli"), sobre la terminación "tepec" (monte), y estos dos pictogramas-ideogramas dan la lectura fonética de la palabra y su primer significado, "loma de los perros". El nivel ulterior de interpretación proporciona dos significados metafórico-simbólicos: el primero deriva de Ixcuina, "La Flechadora", un principio sagrado/diosa originario de la cultura huaxteca que "tuvo un simbolismo fundamentalmente relacionado a la luna y sus ciclos astronómicos, a la fertilidad de la tierra y a la fecundidad femenina, entre otros aspectos." (Rincón Huarota, 2009). En el panteón religioso sincrético mexica Ixcuina se identifica con Tlazolteotl ("Devoradora de la Suciedad", "Comedora de Inmundicias"), diosa relacionada con la tierra, la carnalidad, el parto, la luna, imagen vinculada con principios femeninos duales, a la vez de bien y mal, como enfermedad y curación, transgresiones y absolución, protectora de los médicos. IzcuinaTlazolteotl toma su fonético de "itzcuintli", significando entonces nuestro glifo "monte o lugar consagrado a Izcuina". "Itzcuintli" puede también referirse, por extensión, a "itzcuin patli" ("patli" siendo "medicina", "medicamento" - (Siméon, 1977:377), la "yerba del perro" (Solanum schlechtendalianum, Solanaceae), un arbusto medicinal nativo de Mesoamérica, todavía utilizado en Veracruz y Oaxaca como contra la infección en la mordedura del crótalo ${ }^{16}$, significando entonces "cerro o lugar del itzcuin patli" o "cerro o lugar de la yerba del perro" ${ }^{17}$. En los tres significados, el fonético y los dos

15. El volumen de Orozco y Berra, Historia Antigua y de la Conquista de México, es consultable en su versión electrónica en la Biblioteca Digital UNAM, Universidad Autónoma de México, http://dgb.unam.mx/.

16. Biblioteca Digital de la Medicina Tradicional Mexicana, Universidad Nacional Autónoma de México, http://www.medicinatradicionalmexicana.unam.mx/monografia. php? $1=3 \& \mathrm{t}=\& \mathrm{id}=7579$.

17. Escribe Peñafiel: "Itzcuintepec. -- Itzcuin tepec. -- Itzcuintepec.Un perro con manchas negras, itzcuintli, sobre la terminación tepec, da la lectura fonética de la 
metafóricos, es notable la integración de lo natural (un animal que simboliza una planta) y lo religioso-teológico (un animal que simboliza una diosa), ambos con atributos e influencias sobre lo humano. Es más, los dioses mesoamericanos, antropomórficos y con cualidades básicamente humanas, son productos del medio social donde surgieron (Florescano, 1997). Los dioses creadores, invisibles e impalpables, sin embargo están representados a menudo con una mezcla de rasgos humanos, vegetales y animales, en oposición entre lo uno y lo múltiple, en donde lo humano conjuga las múltiples manifestaciones de lo divino y lo natural. De esta manera, a través de metonimias y/o metáforas, "el cuerpo único del dios, que es siempre el cuerpo humano, se transforma en las «mil formas» o en los «cuatrocientos cuerpos del dios», las cuales no son otra cosa más que proyecciones del cuerpo humano en el mundo natural o animal” (p. 51-58).

El último glifo, Acamiltzinco (Matrícula, Lám. 27, fig.20), es un ejemplo de escritura polisilábica: especificando lo que escribe Peñafiel y que aparece en el sitio del Project de Berkeley ${ }^{18}$, el pictograma-ideograma representa una caña ("acatl") verde de maíz con una mazorca o una flor roja a su lado derecho y al izquierdo una mazorca amarilla o un jilote (el maíz tierno), puesta sobre el signo que representa la tierra cultivada, el campo labrado ("milli" 19 - en el español contemporáneo de México "milpa", derivado de "milli" con el sufijo "-pa", "hacia", es todavía hoy el nombre común para

palabra, pero no su significado. Ixcuina, diosa de la sensualidad, toma su fonético de itzcuintli; "lugar consagrado á Ixcuina;" la palabra Itzcuintepec puede significar tambien "lugar del itzcuin patli, ó yerba del perro."” http://oldweb.geog.berkeley. edu/ProjectsResources/Glyphs/Plate15/Itzcuintepec.html.

18. Acamiltzinco. -- Aca-mil-tzinco. -- Acamilcinco. Una caña verde con flor roja ó gilote, y un fruto amarillo ó mazorca, sobre el signo milli, heredad ó tierra cultivada, y la terminación tzinco expresada por la mitad inferior del cuerpo humano, producen esta escritura polisilábica completa. Amilli significa tierra de regadío, de milli, tierra, y de atl, agua; pero no es esta la radical de Acamiltzinco, diminutivo que significa milpillas, hoy nombre vulgar de los pequeños sembrados de maíz: esta interpretación parece más probable por ser el jeroglífico parecido en sus primeras radicales al de Acamilixtlahuacan. http://oldweb.geog.berkeley.edu/ ProjectsResources/Glyphs/Plate01/Acamiltzinco.html.

19. "Milli”, http://www.tetlacuilolli.org.mx/glifos.php?id_comp_glifico=1234983440\&act $=$ ant\&ord_glifo=1236647938. 
indicar el campo de maíz de cultivo tradicional o de monocultivo- ver §2); la partícula diminutiva "tzintli", que literalmente significa "base, ano" 20 y el sufijo "-c", “-co", que indica "lugar”21, ambos expresados fonéticamente por el glifo que representa la parte inferior de un ser humano masculino en cuclillas 22 . "Acamiltzinco" (que no fue posible ubicar en un mapa actual) es así "lugar de los pequeños campos de maíz". En este caso también es considerable la estilización realística de la naturaleza, el paisaje y el cuerpo humano, y al mismo tiempo su compenetración y su uso metafórico. No es explícita la presencia de dioses, pero el maíz siempre es parte integrante de la ontología de los dioses, del ser humano, de la naturaleza y del mundo en general.

\section{Conclusiones}

Este ensayo es un primer intento de análisis de casos de integración de los espacios humanos, naturales y divinos en la expresión comunicativa semiótica y multitextual de los pictogramas de la cultura nahuatl del México prehispánico desde el punto de vista ecolingüístico, integrado con el concepto de cosmoteandrismo. Las preguntas centrales de este análisis pueden contestarse de la manera siguiente: 1. el discurso de las poblaciones de habla nahuatl prehispánico abarca muchos niveles, formas lingüísticas, expresivas, semánticas y semióticas, y es extremadamente entretejido con su cosmovisión, la cual es también increíblemente compleja e intricada, definiendo una Weltanschauung muy articulada y diferente de la occidental - que a su lado parece hasta maniquea en

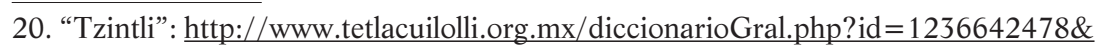
$\underline{\mathrm{ctr}}=$ nah\&busq $=$ tzintli.

21. Ver también http://www.columbia.edu/cu/lweb/digital/collections/cul/texts/ ldpd_6072319_000/pages/ldpd_6072319_000_00000006.html.

22. Véase la nota 7, y también el diccionario nahuatl prehispánico de los códices del Fondo Mexicano de la Biblioteca Nacional de Francia, parte del proyecto digital interactivo del CIESAS -ver nota 15-, denominado Amoxcalli (la Casa de los Libros), en la página http://www.amoxcalli.org.mx/glifos.php?id codice $=0896 \&$ id lamina $=089 \_6$ 1\&id_zona $=089 \_6 \_1 \_C \& i d \_c o m p=089 \_6 \_1 \_C \_45 \&$ id glifo $=089 \_6 \_\overline{1}$ C $\_45 \_09.09 .09$. 
su separación entre lo humano y lo no - humano (natural). 2. Había en la cosmogonía mexica una profunda interrelación, que llamaremos cosmoteándrica, entre las dimensiones teológica, antropológica y ecológica-natural, que en las últimas décadas muchos investigadores han tratado de analizar bajo una perspectiva siempre más amplia y exhaustiva. Los aportes de la ecolingüística contribuyen a profundizar y unificar todavía más los varios enfoques contiguos. 3. Sí, hay elementos que podrían aplicarse hoy para extender la ecosofía y para un discurso global más biocéntrico, haciendo hincapié en la unión medular de los planes de la tierra, del inframundo y del cielo, que necesariamente hay que rescatar como parte de las "metaphors we need to live by", tras investigar las que nos sustentan la vida, la visión y la muerte (Lakoff, Johnson, 1980; Goatly, 1996). Como escribe López Austin a propósito de las últimas investigaciones del americanista español J. Alcina Franch, "si el lenguaje y las artes visuales son formas de expresión, y si la lengua náhuatl clásica se caracteriza por la riqueza de sus metáforas, es pertinente buscar en las artes visuales la correlación metafórica de la lengua." (2003:144), tomando además en cuenta el hecho de que las fronteras extremadamente efímeras entre la expresión visual (artística) y la oral/pictórica/ideográfica/fonética/escrita de la lengua nahuatl, son un perfecto ejemplo de como los estudios tradicionales de lingüística y de comunicación han atomizado de manera contraproducente lo que realmente es un unicum multitextual y un proceso unitario e indivisible de significación.

Asimismo, el análisis ecolingüístico del "difrasismo" en las asociaciones de elementos "naturales", "culturales" y "divinos", es muy prometedor. El difrasismo es típico, aunque no exclusivo, del maya y nahuatl clásicos. Consiste en "expresar una misma idea por medio de dos vocablos que se completan en el sentido, ya por ser sinónimos ya por ser adyacentes.” (p. 112; Montes de Oca Vega, 2008:226-228). Lo anterior está respaldado por la sugerencia de López Austin (2003) de que "trascendiendo los ámbitos lingüísticos ...en el análisis de los difrasismos debe tomarse en cuenta la cosmovisión": podemos afirmas que entre ambos aspectos hay retroalimentación recíproca (feedback). Todas estas sugerencias tendrán que desarrollarse y profundizarse 
en un futuro cercano, ya que la ecolingüística necesita quizás más investigaciones acerca de alternativas propositivas y constructivas, que análisis de ecocrítica.

Finalmente, es imprescindible enfatizar una vez más que la perspectiva de integración de la ecolingüística, también como "lingüística ecosistémica" (Couto ${ }^{23}$ ), con todas las ciencias "humanas" y "naturales" ${ }^{24}$, y con la de todos los seres del planeta Gaia, pasa también por la búsqueda y el uso de lenguajes e imaginerías que se originen en visiones del mundo no eurocéntricas, y por ende, no perjudicadas por el dualismo usualmente destructor de las lenguas Standard Average European (Whorf, 2000), exportadas y utilizadas desde la época colonial. Esta ecolingüística "híbrida" quiere reflejar la multiplicidad y la complejidad de los objetos y los contextos que pueden observarse y de los que se pueden traer ejemplos aprovechables y adaptables. No solamente en lo que pertenece a las áreas de habla inglesa - si bien el inglés es hoy la lengua franca de todos los pueblos globalizados del planeta -, hay que acordarnos que "English language patterns encourage the tendency to perceive resources in isolation rather than holistically. Scientists are aware of this tendency to fragment reality" (Chawla, 1991: 253). Es imprescindible abrirse y abrir las investigaciones a otros mundos cognitivos, discursivos y pragmáticos, ya que "it is through mental, social, and physical analogies and metaphors that we interpret ourselves and our environments. ... From our dialectical and ecological point of view it is the case (i) that every level, field and agent interact and are interdependent, (ii) that every agent, field or level change, and (iii) that because the world and nature consist of complex system then new states, relations, and processes emerge all the time. In order both to survive and live a good life individually and as a species we have to develop healthy analogies and metaphors." (Bay, Døør \& Steffensen, 2003:40-42).

23. http://www.meioambienteelinguagem.blogspot.com.br/; pronto será editado un libro acerca de esta teoría innovadora.

24. En cuanto a las disciplinas de las Ciencias Naturales y Biológicas, uno de los muy pocos ensayos de perspectiva integrada, cross-disciplinaria y que abarca también estudios de culturas no europeas e intentos de análisis lingüísticos es el de Zunino, M. y Colomba (1997). 
Con este tipo de investigaciones vislumbramos, para todo estudio y formación en la época actual, de complejidad e incertidumbre, lo que Gagliasso (2010:17) define la ruptura del ideal formal que actúa en dos tiempos, primeramente en la fase de la ideación. Es preciso desprenderse de las dinámicas usuales de los lenguajes propia de la etapa ideacional de la investigación: siendo ésta el lugar de procesos mentales - personales y colectivos - que construyen el acceso epistémico a lo nuevo, necesita de alteraciones radicales, extensiones y enlaces interdisciplinarios imprevistos e innovadores.

Recebido: 30/07/2012

Aceito: 12/11/2012

francesca.zunino@unimore.it

\section{Referencias bibliográficas}

AINSA, F. Del topos al logos. Propuestas de geopoética. Madrid-Frankfurt am Main: Vervuert, [1976] 2006

ALEXANDER, R. Framing Discourse on the Environment. A Critical Discourse Analysis Approach. Londres: Routledge, 2009

AMITH, J.D. Enciclopedia Cultural Náhuatl: Botánica y Zoología, Río Balsas, Guerrero. FAMSI, Foundation for the Advancement of Mesoamerican Studies, Inc., 2007, pp.1-74, solo edición digital, www.famsi.org/ reports/03049es/03049esAmith01.pdf

ASTURIAS, M.A. Hombres de maíz. Madrid: Alianza Editorial, [1949] 1972

BANG, J.C. The ecology of communicative competence. In: A. FILL; H. PENZ (org.) Sustaining language: essays in Applied Ecolinguistics, 2007, pp. 251-265. Tübingen:LIT Verlag.

BANG, J.C.; DØØR, J. Eco-linguistics: a framework. In: AILA 1993: 31-40. Edición digital,

http://www.jcbang.dk/main/ecolinguistics/Ecoling_AFramework1993.pdf

BANG, J. C.; DØØR, J. Dialectics, Ecology, and Order. In: B. KETTEMANN; H. PENZ (org.) ECOnstructing language, nature and society: the ecolinguistic 
project revisited; essays in honour of Alwin Fill. Tübingen : Stauffenburg, 2000: 49-62

BANG, J.C.; DØØR, J.; NASH, J.; STEFFENSEN, S.V. Language, ecology and society: a dialectical approach. Londres: Continuum, 2008

BARLOW, R. H. The Extent of the Empire of the Culhua Mexica. Berkeley, University of California Press, 1949

BARTHES, R. Rhétorique de l'image. Communications, 4 : 40-51, 1964

BARTRA, A. Del teocintle a los corn pops. In: G. ESTEVA; C. MARIELLE (org.) Sin maíz no hay país. México, DF: Conaculta, 2003, pp. 219-249

BASTARDAS i BOADA, A. Linguistic sustainability and language ecology. Journal of Language \& Ecology, 1,4, 2005, solo edición digital, http://www. ecoling.net/Sustainability.pdf

- Complejidad y emergencia en lingüística y ciencias de la comunicación. Glossa. An Ambilingual Interdisciplinary Journal, 4,2: 312 330, 2009. Edición digital, http://bibliotecavirtualut.suagm.edu/Glossa2/ Journal/Oct2009/Complejidad-y-Emergencia.pdf

BAY D.; DØØR J.; STEFFENSEN, S.V. Modality, ecology, metaphor. metaphorik.de, 4, 2003, solo edición digital, http://www.metaphorik.de/04/ baydoorsteffensen.htm

BOFF, L. Ecologia, mondialità, mistica. Assisi: Cittadella Editore, 1993

BOWERS, C.A. Philosophy, language and the Titanic mind-set. Language \& Ecology, 2, 1, 2007, solo edición digital, http://www.ecoling.net/ titanic.pdf

BRODA, J. Simbolismo de los volcanes. Los volcanes en la cosmovisión mexicana. Arqueología Mexicana, XVI, 95: 40-47, 2009.

BRODA, J.; IWANISZEWSKI. S.; MONTERO GARCÍA, I.A. La montaña en el paisaje ritual. México, DF: UNAM, 2001

CARRASCO, D. Cosmic Jaws: We Eat the Gods and the Gods Eat Us. Journal of the American Academy of Religion, 63, 3: 429-463, 1995

CASILLAS C.; LETICIA E.; VARGAS, L. A. La alimentación entre los mexicas. In: F. MARTÍNEZ CORTÉS (org.) Historia general de la medicina en México, Tomo 1. México, DF: UNAM, 1984, pp. 13-156

CHAWLA, S. Linguistic and philosophical roots of our environmental crisis. Environmental Ethics, 13/3:153-173, 1991 
COE, M. D. Mexico. From the Olmecs to the Aztecs. Londres: Thames and Hudson, [1962] 1994

COOKE, R. Human settlement of Central America and northernmost South America (14,000-8000 BP). Quaternary International, 49/50:177-190, 1998

COUTO, H.H. do. Ecolingüística. Estudio das relações entre língua e meio ambiente. Brasília: Thesaurus, 2007

Ecological approaches in linguistics: a historical overview. Language sciences, Special Issue, Ecolinguistics: the Ecology of Language and Science, en prensa

DABROWSKA, K.M. El concepto de ilhuicatl en la cosmovisión nahua y sus representaciones gráficas en códices. Revista española de antropología americana, 38, 2:151-171, 2008

DILLEHAY, T.D. The Settlement of the Americas: A New Prehistory. New York: Basic Books, 2000

DÖRING, M., 2003. The Politics of Nature. Constructing the German Reunification During the Great Odra Flood 1997. Environment and History, 9: $195-214$

DULL, R. The maize revolution: a view from El Salvador. In: J. Staller; R. TYKOT; B. BENZ (org.) Histories of maize. Oxford: Elsevier, 2006, pp. 357 366

DULL, R. Unpacking El Salvador's ecological predicament: Theoretical templates and "long-view" ecologies. Global Environmental Change, 18: 319-329, 2008

Fairclough, N. Language and power. Harlow: Pearson Education Limited, 2001

Routledge, 2003

Analyzing discourse: textual analysis for social research. Londres:

FAUCONNIER, G.; TURNER, M. The way we think. Conceptual blendings and the mind's hidden complexities. New York: Basic Books, 2002

FERNÁNDEZ, A. Diccionario ritual de voces nahuas. México, DF: Panorama Editorial, 1992

FILL, A. The language impact. Evolution-System-Discourse. Londres: Equinox, 2010 
FILL, A.; MÜHLHÄUSLER, P. (org.) The ecolinguistic reader. Language, ecology and environment. Londres: Continuum, 2001

FLORESCANO, E. Sobre la naturaleza de los dioses de Mesoamérica. Estudios de cultura Náhuatl, 27: 41-67, 1997

GAGLIASSO, E. Introduzione. Tra epistemologia e vissuto: il ruolo delle metafore del vivente. In: E. GAGLIASSO; G. FREZZA (org.) Metafore del vivente. Linguaggi e ricerca scientifica tra filosofia, bios e psiche. Milano: Franco Angeli, 2010, pp. 17-25

GARCÍA COOK; A. Richard Stockton MacNeish y el origen de la agricultura. Arqueología Mexicana, V, 25: 40-43, 1997

GLOTFELTY, C.; FROMM, H. The ecocriticism reader: landmarks in literary ecology. Athens: University of Georgia Press, 1996

GOATLY, A. Green grammar and grammatial metaphor, or language and myth of power, or metaphors we die by. Journal of Pragmatics, 25: 537-560, 1996

HALFFTER, G. La diversidad biológica de Iberomérica. Acta Zoologica Mexicana, Volumen Especial, 1992

HALLYDAY, M.A.K. New ways of meaning. The challenge to Applied Linguistics. In: A. FILL; P. MÜHLHÄUSLER (org.) The ecolinguistic reader. Londres: Continuum, 2001

HASLER HANGERT, A. Manual de gramática del nahuatl moderno. Tlaxcala: Universidad Autónoma de Tlaxcala, 1995

HARRÉ, R.; BROCKMEIER, J.; MÜHLHÄUSLER, P. Greenspeak. A Study of Environmental Discourse. Londres: Sage, 1999

HERMANNS, F. Sprachgeschichte als Mentalitätsgechichte. Überlegungen zu Sinn und Form und Gegenstand historischer Semantik. In: A. GARD; K. MATTHEIER; O. REICHMANN (org.) Sprachgeschichte des Neuhochdeutschen. Tübingen: Stauffenburg, 1995, pp. 69-101

JÄGER, S. Discourse and Knowledge: Theoretical and Methodological Aspects of a Critical Discourse and Dispositive Analysis. In: R. WODAK; M. MEYER (org.) Methods of Critical Discourse Analysis, Londres: Sage International, 2001, pp. 33-62

JOHANSSON KERAUDREN, P. La imagen en los códices nahuas: consideraciones semiológicas. Estudios de Cultura Náhuatl, 32: 69-124, 2001. 
JUNG, M. Ecological criticism of language. In: A. FILL ; P.MÜHLHÄUSLER (org.), 2001, pp.270-285

KLEIN, C. F. (2004) La iconografía y el arte mesoamericano. Arqueología Mexicana, X, 55: 28-35, 2004

LAKOFF, G.; JOHNSON, M. Metaphors we live by. Chicago: University of Chicago Press, 1980

LATOUR, B. Wir sind nie modern gewesen. Versuch einer symmetrischen Anthropologie. Frankfurt am Main: Fischer, 1998

LEÓN-PORTILLA, M. La filosofía náhuatl estudiada en sus fuentes. México, DF: Instituto Indigenista Interamericano, 1956

Los nombres de lugar en náhuatl. Su morfología, sintaxis y representación glífica. Estudios de Cultura Náhuatl, 15: 37-72, 1982

- La multilingüe toponimia de México: sus estratos milenarios. Philologica hispaniensia: in honorem Manuel Alvar, 1: 347-360, 1983

33, 2009

Toponimia e Identidad. Arqueología Mexicana, XVII, no.100: 28 -

LONG-SOLÍS, J.; VARGAS, L-A. Food Culture in Mexico. Westport, Ct.: Greenwood publishing Group, 2005

LÓPEZ AUSTIN, A. El árbol cósmico en la tradición mesoamericana. Monografías del Jardín Botánico de Córdoba, 5: 85-98, 1997

Difrasismos, cosmovisión e iconografía. Revista española de antropología americana, , 1: 143-160, 2003

. Mitos e íconos de la ruptura del eje cósmico: un glifo toponímico de las piedras de Tízoc y del ex-arzobispado. Anales del Instituto de Investigaciones Estéticas, 89: 93-134, 2006.

. Estudio acerca del método de investigación de fray Bernardino de Sahagún. Estudios de Cultura Náhuatl, 42:353-400, 2011.

MACAZAGA ORDOÑO, C. Diccionario de la lengua nahuatl. Con términos, expresiones e inflexiones verbales de la gramática de Carochi-Paredes. México, DF: Editorial Innovación, 1979

MANN, C. 1491. The Americas before Columbus. Londres: Granta Publications, 2005

Martínez. J.L. Hernán Cortés. Versión abreviada. México, DF: FCE, 1992. 
MC CLUNG DE TAPIA, E. La domesticación del maíz. Arqueología Mexicana, V. 25: 34-39, 1997

MENTZ LUNDBERG. B. De árboles, raíces y locativos en la iconografía del México antiguo. Tlalocan, XV: 215-226, 2008

MONTES DE OCA VEGA, M. Los difrasismos en el náhuatl del siglo XVI. México, DF: UNAM, 2000

Los difrasismos: un rasgo del lenguaje ritual. Estudios de cultura

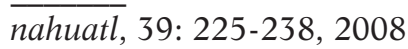

MORIN, E. Introduction à la pensée complexe. Paris: ESF, 1992

MORIN, E. ; MOTTA, R. ; CIURANA, E.R. Éduquer pour l’ère planétaire, la pensée complexe comme méthode d'apprentissage dans l'erreur et l'incertitude humaine. Paris: Balland, 2003

NAESS, A. The shallow and the deep, long range ecology movements: a summary. Oslo: Inquiry, 1973

OROZCO Y BERRA. M. Historia antigua de la conquista de México. México, DF: Tipografía de Gonzalo Esteva, 1880

ORTEGA PACZKA, R. La diversidad del maíz. In: G. ESTEVA; C. MARIELLE (org.) Sin maíz no hay país. México, DF: Conaculta, 2003, pp. 123-154

PANIKKAR, R. Ecosofía: para una espiritualidad de la tierra. Madrid \& San Pablo: D.L., 1994

PAPST, J. Transdisciplinarity: The Unifying Paradigm of Humanities, Natural and Social Sciences. TRANS, Internet-Zeitschrift für Kulturwissenschaften, 15, 2003, solo edición digital, http://www.inst.at/trans/15Nr/01_6/ papst b $15 . \mathrm{htm}$

PENNYCOOK, A. English and colonialism: origins of a discourse. In: A. PENNYCOOK (org.) The cultural politics of English as an international language. Londres: Longman, 1994, pp. 73-103

PRATT, M. Imperial eyes: travel writing and transculturation. Londres: Routledge, 1992

SIMÉON, R. Diccionario de la lengua nahuatl o mexicana. México, DF: Siglo Veintiuno, 1977

SILVERSTEIN, M. Cultural Prerequisites to Grammatical Analysis. In M. SAVILLE-TROIKE (org.) Linguistics and Anthropology: Georgetown University Round Table on Languages and Linguistics. Georgetown: Georgetown University Press, 1977, pp. 139-151 
VALENZUELA VALDIVIESO, E. ¿Es posible aprender geografía a través de la toponimia? Investigación Universitaria Multidisciplinaria: Revista de Investigación de la Universidad Simón Bolivar, 9,9: 16-26, 2010

VAN LEEUWEN, T.; WODAK, R. Legitimizing immigration control: a discourse-historical analysis. Discourse Studies, 1, 1:83-118, 1999

WHORF, B. Language, thought and reality: selected writing of Benjamin Lee Whorf. Cambridge, MA: MIT Press, [1956], 2000

WODAK, R. The discourse-historical approach. In: R. WODAK; M. MYER (org.) Methods of Critical Discourse Analysis. Londres y New York: Sage, 2001, pp. 63-94

ZUNINO, F. Dalle Indie agli "Indios". La Creazione delle Identità SocioEcologiche Americane ed Europee dopo il 1492. In: M. BONDI; G. BUONANNO; C. GIACOBAZZI; M. D. PANFORTI (org.) DiaLogos. Appartenenze Multiple: Prospettive Interdisciplinari su Immigrazione, Multiculturalismo, Esperienze Cross-Culturali. Módena: Officina Edizioni, 2011, pp. 79-94

Latin American Ecolinguistic Issues and the Global Greening of Discourse: Historical Roots and Present Questions. In: J.C. BANG; A. VIBEKE Lindø; M. DOERING; J. NASH (org.) Ecolinguistics: Taking Stock of an Emerging Paradigm in Linguistics and its State of the Art. Tübingen: Schauffenburg, en prensa. 